\title{
PREVALÊNCIA DO DESCARTE DE BOLSAS DE SANGUE POR SÍFILIS EM UM SERVIÇO DE HEMOTERAPIA NO ESTADO DE SERGIPE, BRASIL
}

\section{PREVALENCE of DISPOSAL OF BLOOD UNITS DUE TO SYPHILIS IN A HEMOTHERAPY SERVICE IN THE STATE OF SERGIPE, BRAZIL}

\author{
Vanessa Oliveira Amorim¹, Ester Benz ${ }^{1}$, Rafael de Souza Aguiar ${ }^{1}$, \\ Cibele Macedo Santos ${ }^{1}$, Íkaro Daniel de Carvalho Barreto², \\ Edivan Rodrigo de Paula Ramos ${ }^{3}$, Marco Aurélio de Oliveira Góes ${ }^{1,4}$
}

\section{RESUMO}

Introdução: A doação de sangue é um ato capaz de salvar vidas. Quando a triagem sorológica é positiva, a bolsa de sangue é descartada. Este trabalho teve como objetivo avaliar a prevalência de descarte de bolsas de sangue em razão da positividade dos testes de triagem para sífilis.

Métodos: Foi realizado um estudo epidemiológico observacional, com avaliação transversal e da tendência temporal da positividade do VDRL de acordo com as variáveis epidemiológicas, a partir de dados coletados no banco de sangue do Hemocentro de Sergipe das doações ocorridas de 1 de janeiro de 2007 a 31 de dezembro de 2018.

Resultados: Do total de 308.953 doações de sangue, 4.594 bolsas $(1,48 \%)$ foram descartadas devido a positividade para sífilis. A positividade foi significativamente maior no sexo masculino $(O R=1,14)$. Houve aumento significativo da prevalência com o aumento da faixa etária, de 0,63\% (IC95\% 0,51-0,760 em menores de 20 anos até 4,54\% (IC95\% 3,88-5,30) em pessoas com 60 anos e mais. Entre o período de 2007-2011 foi identificada uma tendência de crescimento da soroprevalência para sífilis (APC $=31,4$ ) seguida pela estabilização do número de descarte de bolsas nos últimos anos.

Discussão: $O$ estudo identifica que, apesar de ter havido um aumento significativo no período inicial, a tendência foi de estabilidade nos últimos anos avaliados. É importante destacar a falta de um exame que indique concretamente a presença ativa da sífilis, pois todos os testes podem permanecer como cicatrizes por longos períodos, mesmos em pessoas com o tratamento adequado.

Palavras-chave: Hemocentro; Doadores de sangue; Sífilis; Epidemiologia

\section{ABSTRACT}

Introduction: Blood donation is an act that can save lives. When serological screening is positive, the blood bag is discarded. This study aimed to assess the prevalence of blood bag disposal due to positive screening tests for syphilis.

Methods: An observational epidemiological study was carried out, with cross-sectional temporal trend assessment of VDRL positivity according to epidemiological variables, based on data collected in the blood bank of the Sergipe Blood Center, from the donations that occurred from $1^{\text {st }}$ January, 2007 to December $31^{\text {st }}, 2018$.

Results: Of the total of 308,953 blood donations, 4,594 bags (1.48\%) were discarded due to positive serology for syphilis. Positivity was significantly higher in males (odds ratio $=1.14$ ). There was a significant increase in prevalence with age, from $0.63 \%$ $(95 \% \mathrm{Cl} 0.51-0.76)$ in individuals under 20 years old to $4.54 \%(95 \% \mathrm{Cl} 3.88-5.30)$
Clin Biomed Res. 2021;41(2):133-140

1 Departamento de Medicina de Lagarto, Universidade Federal de Sergipe. Lagarto, SE, Brasil.

2 Programa de Pós-Graduação em Biometria e Estatística Aplicada, Universidade Federal Rural de Pernambuco. Recife, PE, Brasil.

3 Universidade Federal do Paraná. Curitiba, PR, Brasil.

4 Diretoria de Vigilância em Saúde, Secretaria de Estado da Saúde de Sergipe. Aracaju, SE, Brasil.

Autor correspondente: Marco Aurélio de Oliveira Góes maogoes@gmail.com Departamento de Medicina, Universidade Federal de Sergipe Rua Cláudio Batista, n 505 49060-676, Aracaju, SE, Brasil. 
Amorim et al.

in those with 60 years and over. Between 2007-2011, a trend of increase in seroprevalence for syphilis was identified (annual percent change $=31.4$ ), followed by stabilization in the disposal of bags in recent years.

Discussion: Despite the increase in syphilis reported in society, the study identified that, although there was a significant increase in the initial period, it has been followed by stability in the last years evaluated. What is important is the lack of a test that in fact indicates the active presence of syphilis, since all tests can identify serological scars that remain for long periods, even in people with proper treatment.

Keywords: Blood center; Blood donors; Syphilis; Epidemiology

\section{INTRODUÇÃO}

A doação de sangue é um ato capaz de salvar vidas ${ }^{1}$. De acordo com a legislação brasileira toda doação deve ser voluntária, altruísta, anônima, não remunerada e com sigilo das informações emitidas ${ }^{2}$. A implementação do uso de hemocomponentes na prática médica ocorre há mais de 50 anos e se mantém até os dias atuais. Trata-se de um produto humano insubstituível. O sangue doado perpassa por várias etapas, desde a coleta até o adequado acondicionamento, cujo processo depende do volume e da disponibilidade de doadores. Este, portanto, é o maior desafio dos hemocentros ${ }^{3}$.

No Brasil, os procedimentos hemoterápicos são regulamentados pelo Ministério da Saúde, por meio da RDC n ${ }^{\circ} 34$, de 11 de junho de 2014 e da portaria de consolidação $n^{\circ} 5$, de 28 de setembro de 2017, com a finalidade de garantir a qualidade dos processos e produtos, minimizar riscos e promover a segurança transfusional ${ }^{2,4}$. Os órgãos responsáveis pela captação de sangue são os hemocentros distribuídos pelo país.

Para a doação de sangue ser concluída com êxito, os indivíduos precisam passar pelas etapas da triagem clínica e sorológica. A triagem clínica consiste numa avaliação do estado de saúde atual do indivíduo e de suas possíveis exposições consideradas de riscos ${ }^{5}$. Os principais fatores impeditivos para a doação de sangue incluem valores baixos de hemoglobina e hematócrito, hipertensão (no momento da doação) e risco para Infecções Sexualmente Transmissíveis (IST) ${ }^{6}$.

A triagem sorológica ocorre após aptidão na triagem clínica e é realizada com o sangue doado conforme critérios adotados pelo Ministério da Saúde. São aplicados testes laboratoriais de modo a detectar doenças infecciosas passíveis de transmissão sanguínea. As sorologias testadas incluem Sífilis, Doença de Chagas, Hepatite B, Hepatite C, HIV 1 e 2, HTLV I/II e, em regiões endêmicas, acrescentase o teste para Malária. Há ainda a testagem para o citomegalovírus, porém apenas para indivíduos em situações especiais².

No Brasil, nos últimos dez anos, os casos de Sífilis notificados nas suas formas adquirida, congênita ou gestacional tiveram um aumento significativo. Isso leva a hipótese de que o número de doadores de sangue cujas bolsas foram descartadas por inaptidão sorológica por Sífilis também tenha aumentado. Além disso, tendo em vista que os marcadores sorológicos para Sífilis podem permanecer por toda a vida, é possível admitir que o número de doadores de sangue aptos pode estar comprometido mesmo após tratamento adequado para essa $\mathrm{IST}^{7}$. Neste sentido, este trabalho teve por objetivo determinar e caracterizar o perfil epidemiológico da prevalência de bolsas de sangue descartadas por sorologia positiva para Sífilis no Hemocentro de Sergipe (2007-2018).

\section{MATERIAIS E MÉTODOS}

Trata-se de um estudo epidemiológico observacional retrospectivo, em que foram coletadas informações do banco de dados do hemocentro do estado de Sergipe (HEMOSE). Esta é a única instituição pública sergipana que conta com cinco agências transfusionais. O HEMOSE é responsável por gerir os serviços que vão desde a coleta até o processo de transfusão de sangue regido segundo critérios da Agência Nacional de Vigilância Sanitária (ANVISA).

Neste trabalho foram contabilizadas as doações de sangue ocorridas entre $1^{\circ}$ de janeiro de 2007 e 31 de dezembro de 2018. Os dados tabulados incluíram o total de doações e as variáveis epidemiológicas: ano de doação, sexo, faixa etária (menores de 20 anos, entre 20-29 anos, 30-39 anos, 40-49 anos, 50-59 anos e maiores de 60 anos), tipo de doador (voluntário, reposição, convocado, campanha e autólogo) e regional de saúde (Aracaju, Socorro, Estância, Glória, Itabaiana, Lagarto, Propriá e outro estado).

Os doadores foram classificados como: voluntários (feita por pessoas com objetivo de manter o estoque de sangue através da busca espontânea ao hemocentro), de campanha (coletas feitas para aumentar o estoque de sangue através de campanhas e com coleta fora do hemocentro), de reposição (doação para um determinado paciente que já recebeu ou deverá receber a transfusão), convocado (doador cadastrado que é convidado para doação através do contato do hemocentro) e autólogo (doação realizada pelo paciente para o seu próprio uso).

Apesar do diagnóstico de Sífilis levar em consideração aspectos clínicos, epidemiológicos e história de tratamento prévio da doença associados a testes laboratoriais treponêmicos como o VDLRL 
(Venereal Disease Research Laboratory), a bolsa de sangue é descartada quando um único teste sorológico tem resultado positivo. Sendo assim, esta pesquisa considerou a bolsa de sangue descartada por Sífilis quando o resultado qualitativo do VDRL foi positivo, independente do resultado de uma nova testagem para doença por metodologia diferente.

Os resultados foram analisados, estatisticamente, para as variáveis (sexo, faixa etária, tipo de doador e regional de saúde) em relação à positividade para VDRL, por meio de frequências absolutas/relativas, intervalo de confiança para Odds Ratio (OR) e o teste de associação Qui-quadrado, considerando um nível de significância de $5 \%(p<0,05)$, com auxílio do programa R core team 2020.

Para análise de tendência foram calculadas taxas anuais de prevalência da positividade do VDRL para as variáveis dependentes (sexo, faixa etária e regional de saúde), sendo os anos do período de estudo as variáveis independentes. Foram estimadas as variações anuais percentuais (APC) de uma regressão linear segmentada (jointpont regression). A regressão de Poisson foi utilizada para determinar o número de segmentos necessários para explicar adequadamente a relação entre as duas variáveis, para escolha dos modelos foram considerados os pontos de alteração da tendência que apresentavam nível de significância de $5 \%(p<0,05)$. As tendências foram consideradas estacionárias quando a
APC não foi significativamente diferente de zero $(p>0,05)$, ascendentes quando o coeficiente foi positivo e descendentes quando o coeficiente foi negativo. Para estas análises utilizou-se o software Joinpoint, versão 4.0.4 (Surveillance Research, National Cancer Institute, USA), com acesso gratuito (http://surveillance.cancer.gov/joinpoint/).

O presente trabalho foi desenvolvido conforme diretrizes e normas regulamentadoras de pesquisas envolvendo seres humanos, definida na Resolução $n^{\circ} 196$, de 10 de outubro de 1996, do Conselho Nacional de Saúde. Foi aprovado pelo Comitê de Ética e Pesquisa da Universidade Federal de Sergipe sob o número C.A.A.E 79342917.5.0000.5546.

\section{RESULTADOS}

No período de $1^{\circ}$ de janeiro de 2007 a 31 de dezembro de 2018 houve um total de 308.953 doações de sangue no HEMOSE, com uma média de 25.746 doações anuais. Do total, 16.828 (5,45\%) das bolsas foram descartadas, sendo 4.594 delas por Sífilis $(1,48 \%$ do total de doações). Na maior parte do período avaliado (2007-2018) avaliados não houve diferença estatisticamente significante entre os sexos, embora em 2011, 2016 e na análise do período total, a prevalência foi significativamente maior no sexo masculino (Tabela 1).

Tabela 1: Frequência absoluta e percentual dos doadores cujas bolsas de sangue foram descartadas por sorologia positiva para Sífilis em função do sexo (2007-2018).

\begin{tabular}{cccccccccccc}
\hline \multirow{2}{*}{ ANO } & \multicolumn{3}{c}{ Masculino } & \multicolumn{3}{c}{ Feminino } & \multicolumn{4}{c}{ TOTAL } \\
\cline { 2 - 5 } & VDRL reagente & \% & Doações & VDRL reagente & \% & Doações & VDRL reagente & \% & p-valor \\
\hline $\mathbf{2 0 0 7}$ & 21753 & 215 & 0,98 & 4727 & 48 & 1,01 & 26480 & 263 & 0,99 & 0,530 \\
$\mathbf{2 0 0 8}$ & 23060 & 195 & 0,84 & 5919 & 44 & 0,74 & 28979 & 239 & 0,82 & 0,221 \\
$\mathbf{2 0 0 9}$ & 20887 & 156 & 0,74 & 5187 & 35 & 0,67 & 26074 & 191 & 0,73 & 0,298 \\
$\mathbf{2 0 1 0}$ & 20135 & 455 & 2,25 & 5008 & 90 & 1,79 & 25143 & 545 & 2,16 & 0,055 \\
$\mathbf{2 0 1 1}$ & 20003 & 574 & 2,86 & 6227 & 146 & 2,34 & 26230 & 720 & 2,74 & 0,034 \\
$\mathbf{2 0 1 2}$ & 18611 & 381 & 2,04 & 5854 & 105 & 1,79 & 24465 & 486 & 1,98 & 0,256 \\
$\mathbf{2 0 1 3}$ & 19278 & 282 & 1,46 & 6606 & 86 & 1,3 & 25884 & 368 & 1,42 & 0,379 \\
$\mathbf{2 0 1 4}$ & 19013 & 328 & 1,72 & 7217 & 100 & 1,38 & 26230 & 428 & 1,63 & 0,064 \\
$\mathbf{2 0 1 5}$ & 17683 & 205 & 1,15 & 6863 & 73 & 1,06 & 24546 & 278 & 1,13 & 0,575 \\
$\mathbf{2 0 1 6}$ & 17656 & 282 & 1,59 & 7508 & 93 & 1,23 & 25164 & 375 & 1,49 & 0,039 \\
$\mathbf{2 0 1 7}$ & 16430 & 248 & 1,5 & 7552 & 99 & 1,31 & 23982 & 347 & 1,44 & 0,262 \\
$\mathbf{2 0 1 8}$ & 16632 & 224 & 1,34 & 9144 & 130 & 1,42 & 25776 & 354 & 1,37 & 0,666 \\
Total & $\mathbf{2 3 1 1 4 1}$ & $\mathbf{3 5 4 5}$ & $\mathbf{1 , 5 3}$ & $\mathbf{7 7 8 1 2}$ & $\mathbf{1 0 4 9}$ & $\mathbf{1 , 3 4}$ & $\mathbf{3 0 8 9 5 3}$ & $\mathbf{4 5 9 4}$ & $\mathbf{1 , 4 8}<\mathbf{0 , 0 0 1}$ \\
\hline
\end{tabular}

Dentre o total das doações bloqueadas por Sífilis, 3.545 doações $(77,16 \%)$ foram do sexo masculino, com uma prevalência significativamente maior em relação às mulheres $(p<0,001)$. Com relação $a$ faixa etária, houve significativamente, maior uma maior prevalência da positividade para o VDRL, com o aumento da faixa etária do doador (Tabela 2).

Houve diferença significativa da positividade das amostras em relação a regional de residência do doador. Apesar da regional de Aracaju abranger um 
maior contingente de doadores sororreativos, apresentou menor prevalência (1,33\%), enquanto o destaque ocorreu para a regional de Estância (2,45\%) e Propriá $(2,26 \%)$. Quanto ao tipo de doador, a chance de positividade foi significativamente maior entre os de reposição $(O R=1,49)$ e menor entre convocados e voluntários (Tabela 2 )
A prevalência de descartes de bolsas de sangue por sorologia positiva para Sífilis associada a outros marcadores sorológicos (anti-HIV, anti-HBc, HBsAg, anti-HCV e anti-HTLV I/II) foi significativamente maior que os descartes de bolsas por estes marcadores isoladamente (Tabela 3).

Tabela 2: Prevalência de teste triagem (VDRL) reagente para sífilis por variável demográfica dos doadores de sangue no período entre 2007 e 2018.

\begin{tabular}{|c|c|c|c|c|}
\hline \multirow{2}{*}{ Variáveis } & \multicolumn{2}{|c|}{ VDRL reagente } & \multirow{2}{*}{ Doações (\%) } & \multirow{2}{*}{ OR (IC95\%) } \\
\hline & $\mathbf{n}$ & $(\mathrm{IC} 95 \%)$ & & \\
\hline \multicolumn{5}{|l|}{ Sexo } \\
\hline Feminino & 1049 & $1,34(1,26-1,43)$ & $77812(25,19)$ & $0,87(0,81-0,94)^{*}$ \\
\hline Masculino & 3545 & $1,53(1,48-1,58)$ & $231141(74,81)$ & $1,14(1,06-1,22)^{*}$ \\
\hline \multicolumn{5}{|l|}{ Faixa Etária } \\
\hline$<20$ anos & 93 & $0,63(0,51-0,76)$ & $14810(4,79)$ & $0,41(0,33-0,49)^{*}$ \\
\hline 20 a 29 anos & 1127 & $0,96(0,90-1,01)$ & $117892(38,16)$ & $0,55(0,49-0,56)^{*}$ \\
\hline 30 a 39 anos & 1328 & $1,37(1,29-1,44)$ & $97196(31,46)$ & $0,88(0,82-0,94)^{*}$ \\
\hline 40 a 49 anos & 1211 & $2,17(2,05-2,29)$ & $55800(18,06)$ & $1,64(1,53-1,75)^{*}$ \\
\hline 50 a 59 anos & 683 & $2,53(2,34-2,74)$ & $19911(6,44)$ & $2,59(2,28-2,81)^{*}$ \\
\hline 60 anos e mais & 152 & $4,54(3,88-5,30)$ & $3344(1,08)$ & $3,23(2,74-3,81)^{*}$ \\
\hline \multicolumn{5}{|l|}{ Regional de Saúde } \\
\hline Regional Aracaju & 2654 & $1,33(1,28-1,38)$ & $199328(64,52)$ & $0,75(0,71-0,79)^{*}$ \\
\hline Regional Socorro & 779 & $1,73(1,62-1,86)$ & $44898(14,53)$ & $1,20(1,11-1,30)^{*}$ \\
\hline Regional Estância & 317 & $2,45(2,20-2,73)$ & $12916(4,18)$ & $1,71(1,52-1,92)^{*}$ \\
\hline Regional Glória & 97 & $1,59(1,30-1.92)$ & $6104(1,98)$ & $1,07(0,87-1,31)$ \\
\hline Regional Itabaiana & 335 & $1,51(1,36-1,68)$ & $22148(7,17)$ & $1,02(0,91-1,14)$ \\
\hline Regional Lagarto & 193 & $1,58(1,37-1,82)$ & $12216(3,95)$ & $1,07(0,92-1,23)$ \\
\hline Regional Propriá & 137 & $2,26(1,92-2,67)$ & $6042(1,96)$ & $1,55(1,31-1,85)^{*}$ \\
\hline Outro estado & 82 & $1,54(1,24-1,92)$ & $5301(1,72)$ & $1,04(0,83-1,29)$ \\
\hline \multicolumn{5}{|l|}{ Tipo de Doador } \\
\hline Autólogo & 2 & $2,98(0,21-10,86)$ & 65 & $2,04(0,49-8,33)$ \\
\hline Reposição & 3048 & $1,73(1,67-1,79)$ & 173285 & $1,49(1,40-1,58)^{*}$ \\
\hline Convocado & 49 & $0,57(0,43-0,75)$ & 8488 & $0,37(0,28-0,49)^{*}$ \\
\hline Campanha & 188 & $1,35(1,17-1,56)$ & 13716 & $0,90(0,78-1,04)$ \\
\hline Voluntário & 1307 & $1,18(1,12-1,25)$ & 108805 & $0,71(0,67-0,76)^{*}$ \\
\hline Total de Doações & 4594 & $0,82(0,78-0,84)$ & 304359 & \\
\hline
\end{tabular}

IC95\%: intervalo de confiança de 95\%; OR: odds ratio.

Tabela 3: Prevalência dos marcadores de triagem para as doenças infecciosas transmissíveis em doadores de sangue de acordo com o resultado de VDRL, 2007-2018.

\begin{tabular}{lccccc}
\hline \multirow{2}{*}{$\begin{array}{c}\text { Marcadores sorológicos } \\
\text { reagentes }\end{array}$} & \multicolumn{2}{c}{ VDRL reagente $\mathbf{( n = 5 4 9 4 )}$} & VDRL não reagente (304359) & OR (IC5\%) \\
\cline { 2 - 6 } & $\mathbf{N}$ & $\mathbf{\%}$ & $\mathbf{N}$ & \% & \\
\hline Anti-HBC & 134 & 2,4 & 4156 & 1,4 & $5,2(4,7-5,8)^{\star}$ \\
HBsAg & 57 & 1,0 & 1863 & 0,6 & $2,0(1,6-2,7)^{\star}$ \\
Anti-HCV & 64 & 1,2 & 1545 & 0,5 & $2,8(2,2-3,6)^{\star}$ \\
Anti-HTLV I/II & 56 & 1,0 & 653 & 0,2 & $5,7(4,4-7,5)^{\star}$ \\
Anti-HIV & 116 & 2,1 & 2421 & 0,8 & $3,2(2,7-3,9)^{\star}$ \\
\hline
\end{tabular}

IC95\%: intervalo de confiança de 95\%. *valor de $p<0,05$ 
No período avaliado foi observada uma tendência uma inflexão, mudando a tendência no descarte de bolsas reativas para Sífilis, evidenciando uma tendência crescente entre 2007 e 2011 (APC = 31,41), seguida por um período de diminuição não significativa, indicando estabilidade da taxa entre 2011e 2018 (Figura 1).

A análise temporal da taxa de descarte de bolsas de sangue por Sífilis em função das variáveis sóciodemográficas demonstrou tendência de estabilidade para a maioria das variáveis. Contudo, pontos de inflexão foram observados no sexo masculino que apresentou, em um primeiro momento (2007-2011), tendência crescente, mas depois passou a apresentar tendência estável (Tabela 4).

Quanto a faixa etária até 39 anos, as taxas apresentaram tendência estável durante o período. A partir dos 40 anos observa-se a presença de um ponto de inflexão significativo, com tendência decrescente nos últimos períodos. Apenas nas regionais de Aracaju e Propriá ocorreu mudança de tendência durante o período (Tabela 4).

Figura 1: Tendência temporal das taxas da taxa de descarte de bolsas de sangue descartadas por sorologia positiva para Sífilis em Sergipe, 2007 a 2018.

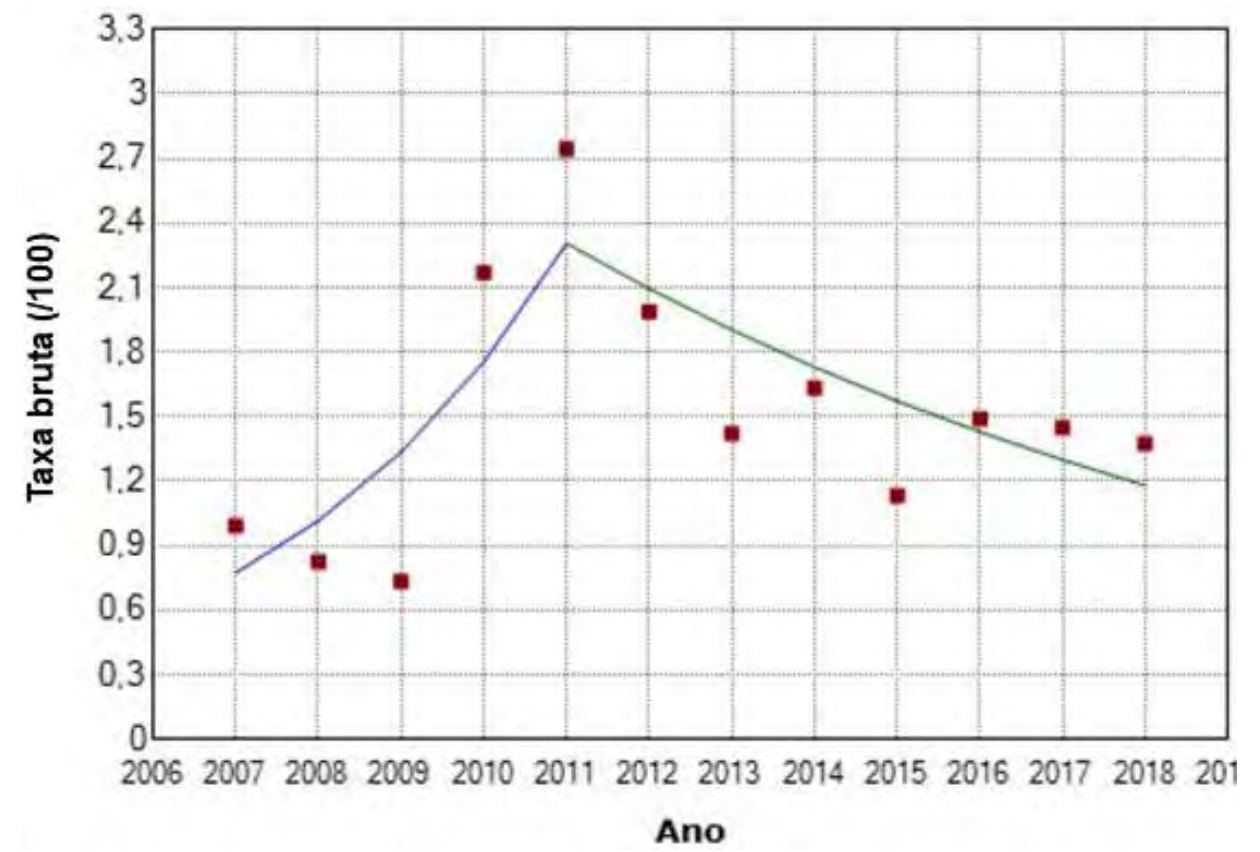

APC: Annual Percent Change. * Indica que a APC é significativamente diferente de zero.

Tabela 4: Análise da tendência temporal do descarte de bolsas de sangue por sorologia positiva para Sífilis em doações de sangue pelas variáveis sexo, faixa etária e regional de saúde (2007-2018).

\begin{tabular}{cccccc}
\hline Variável & Segmento & Período & APC & IC 95\% & Tendência \\
\hline $\begin{array}{c}\text { Sexo } \\
\text { Feminino }\end{array}$ & 1 & & & & \\
Masculino & 1 & $2007-2018$ & 0,04 & $-6,6$ a 6,9 & estável \\
& 2 & $2007-2011$ & $33,14^{\star}$ & 1,9 a 73,9 & crescente \\
Faixa etária & $2011-2018$ & $-9,68$ & 1,3 a $-2,1$ & estável \\
$<20$ anos & 1 & & & $-7,7$ a 8,6 & estável \\
20 a 29 anos & 1 & $2011-2018$ & 0,16 & $-3,6$ a 10,3 & estável \\
30 a 39 anos & 1 & $2012-2018$ & 3,12 & $-6,3$ a 7,6 & estável \\
40 a 49 anos & 1 & $2007-2018$ & 0,46 & $-0,5$ a 82,7 & estável \\
& 2 & $2011-2018$ & $-14,12$ & $-2,0$ a 2,7 & decrescente \\
\hline
\end{tabular}


Amorim et al.

Tabela 4: Continuação

\begin{tabular}{cccccc}
\hline Variável & Segmento & Período & APC & IC 95\% & Tendência \\
\hline 50 a 59 anos & 1 & $2007-2011$ & $55,91^{*}$ & 121,9 a 3,0 & crescente \\
& 2 & $2011-2018$ & $-15,73$ & $-5,5$ a $-3,5$ & decrescente \\
60 anos e mais & 1 & $2007-2012$ & $27,97^{*}$ & 61,5 a 2,5 & crescente \\
& 2 & $2012-2018$ & $-21,57$ & $-9,8$ a $-4,1)$ & decrescente \\
Regional de & & & & \\
Saúde & 1 & $2007-2011$ & $32,5^{*}$ & 0,4 a 74,9 & crescente \\
Aracaju & 2 & $2011-2018$ & $-8,7$ & 1,9 a $-2,0$ & estável \\
& 1 & $2007-2018$ & $-2,04$ & $-9,3$ a 5,8 & estável \\
Estancia & 1 & $2007-2018$ & $-4,3$ & $-13,8$ a 6,1 & estável \\
Itabaiana & 1 & $2007-2018$ & 2,07 & $-4,5$ a 9,1 & estável \\
Lagarto & 1 & $2007-2018$ & $-2,23$ & $-12,2$ a 6,6 & estável \\
Glória & 1 & $2007-2018$ & 2,48 & $-6,9$ a 12,9 & estável \\
Socorro & 1 & $2007-2011$ & $49,4^{*}$ & 14,4 a 95,1 & crescente \\
Propriá & 2 & $2011-2018$ & $-12,2^{*}$ & $-20,3$ a $-3,2$ & decrescente \\
& 1 & & & & \\
\end{tabular}

* Significativo para 0.05 (teste Z, bicaudal). APC: Incremento Percentual Anual (Annual Percent Change); IC 95\%: intervalo de confiança de 95\%.

\section{DISCUSSÃO}

A triagem diagnóstica para Sífilis nos bancos de sangue pode ser realizada com a utilização dos testes sorológicos treponêmicos e não treponêmicos. Contudo, a legislação brasileira não especifica o tipo de teste a ser utilizado. No HEMOSE, o exame realizado no período foi o VDRL, um teste não treponêmico e de alta sensibilidade, mas menor especificidade ${ }^{8}$. Essa variação nos exames utilizados pode interferir nas diferentes taxas encontradas nos diversos estudos.

A prevalência geral da inaptidão sorológica para Sífilis encontrada no presente estudo foi de $1,48 \%$, considerada superior à média nacional registrada em $2017(1,07 \%)$, com destaque para as regiões Nordeste $(1,26 \%)$ e Sul $(1,17 \%)$ do país ${ }^{9}$. O sexo masculino apresentou maior reatividade, assim como os dados encontrados no Brasil para os casos de homens com diagnóstico de Sífilis adquirida $(59,6 \%)^{8}$. Sabe-se, no entanto, que a realidade do hemocentro não retrata a prevalência da infecção na população geral, mas a análise dos fatores epidemiológicos pode contribuir para a garantia e segurança do sangue doado ${ }^{10,11}$.

Em Vera Cruz no México e em Burkina Faso na África Ocidental, foi encontrada uma soroprevalência, em ambas, de $1,5 \%$, semelhante ao presente estudo $^{12,13}$. Enquanto na China a taxa foi inferior, variando nos dois estudos de $0,37 \%$ a $0,97 \%{ }^{14,15}$. A menor prevalência ocorreu no banco de sangue do hospital universitário de Bamako, Mali, com relato de apenas 3 indivíduos infectados $(0,04 \%)^{16}$.

As maiores taxas de reatividade para a Sífilis no Brasil, superior a encontrada neste estudo, foram descritas no Hemonúcleo de Guarapuava
$(2,1 \%)$ e Hemolagos $(2,13 \%)^{17,18}$. Enquanto que taxas inferiores resultaram nos bancos de sangue do Hospital Universitário Polydoro Ernani de São Thiago da Universidade Federal de Santa Catarina $(0,13 \%), \operatorname{HEMOSC}(0,14 \%)$, Centro de Hemoterapia de Caxias do Sul $(0,15 \%)$ e Hemocentro Regional de Cruz Alta $(0,6 \%)^{10,19,20,21}$.

Na maioria dos estudos, a Sífilis foi apontada como segunda maior causa de inelegibilidade sorológica nos bancos de sangue perdendo apenas para a hepatite $B^{1,20,22}$. No entanto, no banco do Serviço de Hemoterapia do Sudoeste, a Sífilis foi considerada a maior causa de bloqueio sorológico $(1,09 \%)^{23}$.

Os resultados apresentados aqui evidenciaram maior soroprevalência no sexo masculino, assim como em outros estudos que variou de 58,3 a $68,8 \%{ }^{17,18,24}$. Uma justificativa apontada por alguns autores é a maior diversidade de parcerias sexuais entre homens, aumentando a exposição e chance de contrair doenças infectocontagiosas ${ }^{23,25}$. Outra possibilidade é o fato de haver maior aptidão masculina para doação de sangue, em detrimento da maior prevalência de anemia pós-menstrual entre as mulheres, o que contraindica a doação ${ }^{11,19,24}$. Além disso, a lei vigente possibilita uma maior frequência de doações entre os homens, sendo 4 por ano, enquanto para as mulheres é permitido apenas 3 doações ${ }^{20}$.

Neste estudo, a maioria dos doadores estava entre 30 e 39 anos (28,91\%), semelhante ao encontrado no Hemocentro Regional de Cruz Alta, cuja maior prevalência ocorreu na faixa dos 31 a 41 anos $(26,7 \%)^{24}$. Enquanto no Hemonúcleo de Guarapuava-PR houve destaque para os indivíduos com uma faixa etária 
maior, ficando entre 41 e 61 anos $(55,7 \%)^{17}$. A maior prevalência entre os indivíduos maiores de 30 anos pode estar relacionada ao estilo de vida adotado que vai desde a prática sexual sem proteção, uso ilícito de drogas injetáveis até a exposição ocupacional| ${ }^{23,26}$.

Quanto ao tipo de doador, dentre as bolsas de sangue descartadas por Sífilis, a mais comum foi referente ao doador de reposição $(66,5 \%)$, seguida dos doadores voluntários $(28,5 \%)$. No entanto, a taxa de maior prevalência ocorreu entre os doadores autólogos (2,9\%). Em geral, doadores de repetição costumam ser mais altruístas e se preocupam com a segurança dos receptores, o que explica menor taxa de bloqueio ${ }^{27}$.

As regionais de saúde do estado de Sergipe apresentaram um maior número absoluto de doadores reativos na capital $(n=2654)$. Contudo, as regionais do interior tiveram maior prevalência. A regional de Estância apresentou 2,45\%, seguido da regional de Propriá com 2,27\%. Essa variação apresentou significância estatística nos resultados. Diferente do encontrado no estudo Baião, Kupek e Petry (2014), cuja variação geográfica não foi significativa ${ }^{19}$.

Durante o período de 2011 a 2016, Jaques, Saldanha e Moraes (2019) relatou um aumento significativo do número de casos reativos para Sífilis $(p=0,003)^{21}$. Diferente do encontrado no presente estudo, em que houve estabilização do número de bolsas descartadas nos últimos anos. Apesar disso, houve elevação na taxa nacional de detecção da Sífilis adquirida que passou de 2,1 para 75,8 casos por 100.000 habitantes de 2010 a $2018^{8}$. Um fator que pode estar relacionado a esse crescimento é a utilização de testes mais sensíveis e específicos por algumas instituições e a baixa disponibilidade do antibiótico para tratamento da Sífilis que ocorreu em meados de 2014 a 2016, o que propiciou um aumento geral do número de casos na população ${ }^{27}$.
A triagem sorológica utilizada no HEMOSE para detecção da Sífilis foi o VDRL, cuja sensibilidade é alta e especificidade é baixa ${ }^{23}$. Na literatura não existe consenso e padronização dos kits utilizados, uma vez que a compra dos produtos depende dos processos de licitação governamental. Diante disso, é possível que haja diferentes taxas de falsos-positivos entre as amostras dos estudos, no entanto não é algo que impossibilita relacioná-los ${ }^{22}$.

A prevalência da positividade para Sífilis apesar da tendência crescente até 2011, passou a apresentar taxas mais baixas nos últimos anos, indicando uma estabilidade, desde então. Esta redução pode ser reflexo dos esforços para a prevenção na comunidade, como o estimula a práticas sexuais mais seguras, mas também às formas de triagem clínica implantadas no hemocentro.

Neste estudo houve uma maior prevalência da inaptidão sorológica por Sífilis em relação a média nacional. Sabe-se que um grande desafio enfrentado atualmente pelos bancos de sangue é a manutenção dos estoques. Desta forma, o conhecimento acerca da prevalência e do perfil epidemiológico dos doadores pode auxiliar os órgãos competentes na utilização de políticas públicas de saúde voltada para a minimização de riscos e melhoria da hemovigilância. Possibilita ainda o diagnóstico de indivíduos infectados, e o adequado tratamento. Além disso, práticas de prevenção podem ser intensificadas de acordo demandas específicas de uma população encontrada em cada regional de saúde.

\section{Agradecimentos}

Os autores agradecem a equipe do HEMOSE pela disponibilização dos dados.

\section{REFERÊNCIAS}

1. Borelli SD, Mazzola JC, Matta ACG, Takemoto AY, Bértoli M. Blood discard rate and the prevalence of infectious and contagious diseases in blood donors from provincial towns of the state of Paraná, Brazil. Rev Bras Hematol Hemoter. 2013;35(6):395-9.

2. Agência Nacional de Vigilância Sanitária (BR). Resolução de Diretoria Colegiada $n^{\circ} 34$, de 11 de junho de 2014. Dispõe sobre as Boas Práticas no Ciclo do Sangue. Diário Oficial da União. 2014 jun 16;1:50.

3. Carlesso L, Santos CF, Guimarães RFS, Silva SL, Viero V, Vieira SV, et al. Estratégias implementadas em hemocentros para aumento da doação de sangue. Rev Bras Promoc Saude. 2017;30(2):213-20.

4. Brasil, Ministério da Saúde. Portaria de Consolidação $n^{\circ} 5$, de 28 de setembro de 2017. Diário Oficial da União. 2017 out 3;Suppl:360.

5. Brasil, Ministério da Saúde, Secretaria de Vigilância em Saúde, Sistema Nacional de Vigilância em Saúde. Caderno de informação: sangue e hemoderivados dados de 2015. Brasília, DF; 2017.

6. Azevedo AS, Nogueira CSC, Artiles CB, Domigues CF, Alves $\mathrm{CN}$, Malheiros GC, et al. Fatores da triagem clínica que impedem a doação de sangue. Rev Cient Fac Med Campos. 2015;10(2):7-11.

7. Brasil, Ministério da Saúde, Secretaria de Vigilância em Saúde, Sistema Nacional de Vigilância em Saúde. Boletim Epidemiológico: Sífilis 2018. Brasília, DF; 2018.

8. Brasil, Ministério da Saúde, Secretaria de Vigilância em Saúde, Sistema Nacional de Vigilância em Saúde, Departamento de Doenças de Condições Crônicas e Infecções Sexualmente Transmissíveis. Indicadores e dados básicos da Sífilis nos Municípios Brasileiros [Internet]. 
2019 [citado em 2019 dez 5]. Disponível em: http://indicadoressifilis. aids.gov.br/.

9. Brasil, Ministério da Saúde, Secretaria de Vigilância em Saúde, Sistema Nacional de Vigilância em Saúde. $6^{\circ}$ Boletim Anual de Produção Hemoterápica: Hemoprod 2017. Brasília, DF; 2018.

10. Martins APB, Silva B, Molin DBD, Mendes GA. Soroprevalência de doenças infecciosas em doadores de sangue do Hemocentro Regional de Cruz Alta-Rio Grande do Sul. Clin Biomed Res. 2015;35(4):211-6

11. Monich AG, Dantas TW, Fávero KB, Almeida PTR, Maluf EC, Capeletto CM, et al. Blood discard rate in a blood center in Curitiba - Brazil. Ten years of study. Transfus Apher Sci. 2017;56(2):130-4.

12. Bisseye C, Sanou M, Nagalo BM, Kiba A, Compaoré TR, Tao I, et al. Epidemiology of Syphilis in regional blood transfusion centres in Burkina Faso, West Africa. Pan Afr Med J. 2013;16:69

13. Balderas NL, Romano JH, Contreras MC, Sarmiento EB, Romano PAH. Trends in prevalence of HIV and syphilis in a central blood bank of Veracruz, Mexico. Transfus Apher Sci. 2019;58(1):94-9.

14. Liu S, Luo L, Xi G, Wan L, Zhong L, Chen X, et al. Seroprevalence and risk factors on Syphilis among blood donors in Chengdu, China, from 2005 to 2017. BMC Infect Dis. 2019;19(1):509.
15. Wu X, Guan Y, Ye J, Fu H, Zhang C, Lan L. Association between syphilis seroprevalence and age among blood donors in Southern China: an observational study from 2014 to 2017. BMJ Open. 2019;9(11): e024393.

16. Jary A, Dienta S, Leducq V, Hingrat QL, Cisse M, Diarra AB, et al. Seroprevalence and risk factors for HIV, HCV, HBV and syphilis among blood donors in Mali. BMC Infect Dis. 2019;19:1064

17. Oliveira VM, Verdasca IC, Monteiro MC. Detecção de sífilis por ensaios de ELISA e VDRL em doadores de sangue do Hemonúcleo de Guarapuava, Estado do Paraná. Rev Soc Bras Med Trop. 2008;41(4):428-30

18. Silva IR, Cardim A. Perfil epidemiológico dos doadores de sangue inaptor por síflis. Rev Enferm Contemp. 2017;6(1):12-9

19. Baião AM, Kupek E, Petry A. Syphilis seroprevalence estimates of Santa Catarina blood donors in 2010. Rev Soc Bras Med Trop. 2014; 47(2):17985.

20. Ril H, Paludo CA, Leite MA, Cattani F. Prevalence of serological ineligibility among blood donors of a hemotherapy center in Caxias do Sul, southern Brazil. Clin Biomed Res. 2017;37(1):11-17.

21. Jaques B, Saldanha PCA, Moraes ACR. Profile of blood donations with a positive serology in Southern Brazil. Hematol Transfus Cell Ther. 2019;42(2):129-33.
22. Silva SMN, Oliveira MB, Martinez EZ. Distribution of serological screening markers at a large hematology and hemotherapy center in Minas Gerais, Southeastern Brazil. Rev Bras Hematol Hemoter. 2016;38(3):206-13.

23. Rebouças KAAF, Narici FM, Santos MN Jr, Neres NSM, Oliveira MV, Souza CL. Seroprevalence of transfusion-transmissible infectious diseases at a hemotherapy service located in southwest Bahia, Brazil. Hematol Transfus Cell Ther. 2019;41(4):324-8.

24. Boff D, Lunkes DS, Kunzler A, Rohr JI. Prevalência de VDRL reagente em doadores do hemocentro regional de Cruz Alta - RS, Brasil, no período de 2003 a 2009. Rev Patol Trop. 2011;40(2):179-84.

25. Almeida Neto C, Murphy EL, McFarland W, Mendrone A Jr, Chen S, Charmone DAF, et al. Profile of blood donors with serologic tests reactive for the presence of syphilis in São Paulo, Brazil. Transfusion. 2009;49(2):330-6.

26. Rodrigues AMX, Barbosa ML, Silva KMR, Santos JS, Costa ACR. Prevalence of major diseases investigated in the serological screening in units of a blood center. ReOnFacema. 2018;4(1):835-40.

27. Pessoni LL, Aquino EC, Alcântara KC. Prevalence and trends in transfusiontransmissible infections among blood donors in Brazil from 2010 to 2016. Hematol Transfus Cell Ther. 2019;41(4):310-5.

Recebido: 18 set, 2020 Aceito: 23 mar, 2021 